\title{
Mapping Local Climate Zones for a Worldwide Database of the Form and Function of Cities
}

\author{
Benjamin Bechtel 1,*, Paul J. Alexander ${ }^{2}$, Jürgen Böhner ${ }^{1}$, Jason Ching ${ }^{3}$, Olaf Conrad ${ }^{1}$, \\ Johannes Feddema ${ }^{4}$, Gerald Mills ${ }^{5}$, Linda See ${ }^{6}$ and Iain Stewart ${ }^{7}$
}

1 Institute of Geography, University of Hamburg, Bundesstraße 55, Hamburg 20146, Germany; E-Mails: juergen.boehner@uni-hamburg.de (J.B.); olaf.conrad@uni-hamburg.de (O.C.)

2 Irish Climate Analysis \& Research Units, Department of Geography, Room 1.8 Laraghbryan House, Maynooth University, Kildare, Ireland; E-Mail: paul.alexander@nuim.ie

3 Institute for the Environment, The University of North Carolina at Chapel Hill, UNC-Chapel Hill, NC 27514, USA; E-Mail: jksching@gmail.com

4 Department of Geography, University of Kansas, 1475 Jayhawk Blvd, Lawrence, KS 66049, USA; E-Mail: feddema@ku.edu

5 School Of Geog, Planning \& Env Policy, Newman Building, UCD, Belfield Dublin 4, University College Dublin, Dublin 4, Ireland; E-Mail: gerald.mills@ucd.ie

6 Ecosystems Services and Management Program, International Institute for Applied Systems Analysis, Schlossplatz 1, Laxenburg 2361, Austria; E-Mail: see@iiasa.ac.at

7 Department of Civil Engineering, University of Toronto, 35 St. George Street, Toronto, ON M5S 2J7, Canada; E-Mail: iain.stewart@utoronto.ca

* Author to whom correspondence should be addressed; E-Mail: benjamin.bechtel@uni-hamburg.de; Tel.: +49-40-42838-4962.

Academic Editor: Wolfgang Kainz

Received: 4 December 2014 / Accepted: 16 January 2015 / Published: 2 February 2015

\begin{abstract}
Progress in urban climate science is severely restricted by the lack of useful information that describes aspects of the form and function of cities at a detailed spatial resolution. To overcome this shortcoming we are initiating an international effort to develop the World Urban Database and Access Portal Tools (WUDAPT) to gather and disseminate this information in a consistent manner for urban areas worldwide. The first step in developing WUDAPT is a description of cities based on the Local Climate Zone (LCZ) scheme, which classifies natural and urban landscapes into categories based on climate-relevant surface properties. This methodology provides a culturally-neutral framework for collecting
\end{abstract}


information about the internal physical structure of cities. Moreover, studies have shown that remote sensing data can be used for supervised LCZ mapping. Mapping of LCZs is complicated because similar LCZs in different regions have dissimilar spectral properties due to differences in vegetation, building materials and other variations in cultural and physical environmental factors. The WUDAPT protocol developed here provides an easy to understand workflow; uses freely available data and software; and can be applied by someone without specialist knowledge in spatial analysis or urban climate science. The paper also provides an example use of the WUDAPT project results.

Keywords: land cover; supervised classification; pixel-based classification; multi-temporal remote sensing; urban climate science; local climate zones; WUDAPT

\section{Introduction}

Consistent knowledge about cities and their internal structure is of high relevance for a number of reasons. Although currently less than 3\% of the Earth's land areas are urban (defined as closely spaced buildings, impervious surfaces and managed outdoor spaces), cities already accommodate most of the world's population, and human activity is concentrated there [1]. On a global scale, urbanisation (that is, the movement of people into cities and the transformation of land cover into urban forms) is proceeding rapidly, which has high impact on the bio-physical surface conditions and resulting energy and material fluxes in urban areas. Urbanisation typically replaces the existing surface cover with impermeable materials and buildings, and concentrates activities, which generate waste disposal into the surrounding air, soil and water. Collectively, cities are one of the main drivers of global environmental change and are also uniquely vulnerable to the consequences of change such as rising sea levels and increased air temperatures. Furthermore, cities produce distinct climatic conditions, which can cause discomfort, heat stress, and exposure to disease and pollution on urban dwellers. In particular, cities are warmer, which is referred to as the urban heat island (UHI) effect [2], as well as more polluted than their rural counterparts.

To study the combined effect of urban climate and climate change on cities and to assess the vulnerability of urban populations, advanced urban models are needed. While a number of useful models already exist, in particular for urban meteorology and chemistry [3], they are often lacking the detailed information about the urban surface that are required as input parameters for these models. In fact, the dearth of data on urban areas has been recently highlighted in the IPCC's 5th Assessment Report on impacts, adaptation and vulnerability of urban areas to climate change [4]. Another problem, until recently, has been the lack of a standard way to characterize cities, which has hampered the ability to collect a consistent set of parameters across the globe. As a result, there have been many studies on individual cities using different nomenclatures but no consistent methodology for comparing these areas at regional or global scales.

In recent years, significant progress has been made in delimiting global high resolution urban land cover masks [5] from multispectral optical and SAR (TerraSAR-X) data [6,7]. Of particular note is the completion of the Global Urban Footprint (GUF) product from the German Aerospace Center (DLR), 
which encompasses worldwide mapping of settlements with an unprecedented spatial resolution of $0.4 \operatorname{arcsec}(\sim 12 \mathrm{~m})$ based on 180,000 scenes from the German radar satellites TerraSAR-X and TanDEM-X [8]. Another promising approach uses imagery from the Visible and Near-Infrared Radiometer of Advanced Spaceborne Thermal Emission and Reflection radiometer (ASTER/VNIR) and an iterative classification process trained with lower resolution urban maps [9]. The alpha version of the global human settlement layer, another global product derived from Landsat data, is now available for four periods between 1975 and 2014 [10]. However, today these global products only provide urban masks, without internal differentiation according to land use and land cover and hence they fall short of providing the data requirements for a number of potential applications in various fields.

There is an urgent need for a comprehensive database on cities world-wide that goes beyond an urban footprint analysis. Such a database would need to capture the internal structure and texture of cities in order to answer a number of important questions about our rapidly urbanizing planet. These include questions on the efficacy of urban-based adaption and mitigation policies in response to climate change and whether actions in one jurisdiction are transferrable to another. These data are also needed for developing and applying climate models, which require detailed descriptions of the urban landscape (at different levels of detail) to simulate both global climate impacts on cities and urban effects on climate. To meet this need the World Urban Database and Access Portal Tools (WUDAPT) project has been created $[3,11,12]$. The objective of WUDAPT is to produce a global high resolution database that captures information on urban form and function, and provides this information in a form suitable for climate science. As part of the WUDAPT initiative, we also need to create the necessary tools, manuals and methodologies to help build this database. This paper represents an attempt to achieve this latter aim.

Development of WUDAPT will require a number of phases, which includes the spatial mapping of appropriate spatial urban types and linking these spatial areas to urban morphology, physical properties and human use. Here, we describe the initial phase of classifying urban areas which can subsequently be linked to additional information and detail in future work.

We present and describe the methodology for deriving a culturally-neutral framework for classifying and delineating urban landscapes into a climatically relevant classification scheme using remote sensing data in combination with local expert-based knowledge and the concept of Local Climate Zones (LCZs). LCZs represent a generic, easily understood, culturally-neutral description of land-use and land-cover (LULC) suited to climate studies [13]. A critical point is that LCZs are universal in their application and they can be linked to measurable urban parameters on urban form and function. The LCZ classification scheme was designed to describe landscapes (urban and natural) that exhibit distinct thermal climates owing to their surface properties and has been used extensively in studies of the urban heat island (UHI). It is ideally suited to our purpose for two reasons. First, it meets the criteria outlined above: it is a universally understood climate-based classification scheme that is based on the physical properties of surfaces, which affect the overlying near surface atmosphere. Second, it has been shown that LCZ types can be identified using different Earth observation data including space-borne optical and thermal data as well as airborne interferometric SAR [14]. Nevertheless, using remote sensing to derive LCZs for cities world-wide is not without challenges. For example, the LCZ scheme is designed to accommodate urban structures from all over the world based only on their climatic properties rather than on local relief, regional or culturally-specific details such as building materials, or natural vegetation types. Such local differences in surface characteristics imply that any regional or culturally specific details are not considered, which 
means that the individual LCZs will have dissimilar spectral properties in different regions, cities, or even within a single city. This makes a supervised classification design which incorporates local expert knowledge of the urban structures pertinent to their city a necessity so that the training data can be gathered in an efficient, accurate, and timely manner. In addition, this process will allow local experts to incorporate potentially important climate factors into their classification scheme.

A protocol for the derivation of LCZ maps for cities is presented here. First, we discuss the appropriateness of LCZ mapping, the lessons learned from previous work and the requirements for implementation; the latter includes decisions on the urban features to be captured, training data, classifiers, and data availability. Based on these considerations, we present a simple and objective mapping method that allows local experts (that is, those with knowledge of a given city) to participate and play an essential role in data collection; this places high demands on the standardization of the process as few local experts will have expertise in climate science, in building and urban studies, or in spatial methodologies (i.e., remote sensing, GIS, and image processing). For this reason, the protocol must be comprised of a simple workflow with publicly available data that can be processed using freely available software by local operators.

\section{Towards a Universal LCZ Mapping Scheme}

In this section, the appropriateness, requirements, and limitations of LCZ mapping methods are discussed as an essential step towards developing the initial global spatial classification on which WUDAPT will be based.

\subsection{Local Climate Zones (LCZs)}

LCZs were recently introduced by Stewart and Oke to standardize the classification of urban and rural field sites for observational UHI studies [13]. Conventionally, the UHI has been defined as an urban-rural air temperature difference at screen height $\left(\Delta \mathrm{T}_{\mathrm{U}-\mathrm{R}}\right)$, but different studies have shown tremendous variety in the landscapes considered as "rural" or "urban" (e.g., airports have served as both urban and rural reference stations) [15]. The LCZ scheme aims to overcome this rather simplistic characterization of the landscape with a universally understood, climate-based classification of urban and rural sites. LCZs are defined as "regions of uniform surface cover, structure, material, and human activity that span hundreds of meters to several kilometers in horizontal scale. Each LCZ has a characteristic screen-height temperature regime that is most apparent over dry surfaces, on calm, clear nights, and in areas of simple relief" [13]. Figure 1 illustrates the 17 standard classes within the LCZ classification. 


\begin{tabular}{|c|c|c|c|}
\hline Built types & Definition & Land cover types & Definition \\
\hline I. Com & $\begin{array}{l}\text { Dense mix of tall buildings to tens of } \\
\text { stories. Few or no trees. Land cover } \\
\text { mostly paved. Concrete, steel, stone, } \\
\text { and glass construction materials. }\end{array}$ & A. Dense trees & $\begin{array}{l}\text { Heavily wooded landscape of } \\
\text { deciduous and/or evergreen trees. } \\
\text { Land cover mostly pervious (low } \\
\text { plants). Zone function is natural } \\
\text { forest, tree cultivation, or urban park. }\end{array}$ \\
\hline 2. Co & $\begin{array}{l}\text { Dense mix of midrise buildings ( } 3-9 \\
\text { stories). Few or no trees. Land cover } \\
\text { mostly paved. Stone, brick, tile, and } \\
\text { concrete construction materials. }\end{array}$ & B. Scattered trees & $\begin{array}{l}\text { Lightly wooded landscape of } \\
\text { deciduous and/or evergreen trees. } \\
\text { Land cover mostly pervious (low } \\
\text { plants). Zone function is natural } \\
\text { forest, tree cultivation, or urban park. }\end{array}$ \\
\hline 3. Com & $\begin{array}{l}\text { Dense mix of low-rise buildings ( } 1-3 \\
\text { stories). Few or no trees. Land cover } \\
\text { mostly paved. Stone, brick, tile, and } \\
\text { concrete construction materials. }\end{array}$ & C. $\mathrm{B}$ & $\begin{array}{l}\text { Open arrangement of bushes, shrubs, } \\
\text { and short, woody trees. Land cover } \\
\text { mostly pervious (bare soil or sand). } \\
\text { Zone function is natural scrubland or } \\
\text { agriculture. }\end{array}$ \\
\hline & $\begin{array}{l}\text { Open arrangement of tall buildings to } \\
\text { tens of stories. Abundance of pervious } \\
\text { land cover (low plants, scattered } \\
\text { trees). Concrete, steel, stone, and } \\
\text { glass construction materials. }\end{array}$ & D. L & $\begin{array}{l}\text { Featureless landscape of grass or } \\
\text { herbaceous plants/crops. Few or } \\
\text { no trees. Zone function is natural } \\
\text { grassland, agriculture, or urban park. }\end{array}$ \\
\hline & $\begin{array}{l}\text { Open arrangement of midrise buildings } \\
\text { (3-9 stories). Abundance of pervious } \\
\text { land cover (low plants, scattered } \\
\text { trees). Concrete, steel, stone, and } \\
\text { glass construction materials. }\end{array}$ & ock or $p$ & $\begin{array}{l}\text { Featureless landscape of rock or } \\
\text { paved cover. Few or no trees or } \\
\text { plants. Zone function is natural desert } \\
\text { (rock) or urban transportation. }\end{array}$ \\
\hline & $\begin{array}{l}\text { Open arrangement of low-rise buildings } \\
\text { ( } 1-3 \text { stories). Abundance of pervious } \\
\text { land cover (low plants, scattered trees). } \\
\text { Wood, brick, stone, tile, and concrete } \\
\text { construction materials. }\end{array}$ & F. Bare & $\begin{array}{l}\text { Featureless landscape of soil or sand } \\
\text { cover. Few or no trees or plants. } \\
\text { Zone function is natural desert or } \\
\text { agriculture. }\end{array}$ \\
\hline 7. Lightweight & $\begin{array}{l}\text { Dense mix of single-story buildings. } \\
\text { Few or no trees. Land cover mostly } \\
\text { hard-packed. Lightweight construction } \\
\text { materials (e.g., wood, thatch, } \\
\text { corrugated metal). }\end{array}$ & G. Wa & $\begin{array}{l}\text { Large, open water bodies such as seas } \\
\text { and lakes, or small bodies such as } \\
\text { rivers, reservoirs, and lagoons. }\end{array}$ \\
\hline & $\begin{array}{l}\text { Open arrangement of large low-rise } \\
\text { buildings ( } 1-3 \text { stories). Few or no } \\
\text { trees. Land cover mostly paved. } \\
\text { Steel, concrete, metal, and stone } \\
\text { construction materials. }\end{array}$ & \multicolumn{2}{|c|}{$\begin{array}{l}\text { VARIABLE LAND COVER PROPERTIES } \\
\text { Variable or ephemeral land cover properties that change } \\
\text { significantly with synoptic weather patterns, agricultural practices, } \\
\text { and/or seasonal cycles. }\end{array}$} \\
\hline 9. Sparsely built & $\begin{array}{l}\text { Sparse arrangement of small or } \\
\text { medium-sized buildings in a natural } \\
\text { setting. Abundance of pervious land } \\
\text { cover (low plants, scattered trees). }\end{array}$ & s. snow cover & $\begin{array}{l}\text { Leafless deciduous trees (e.g., winter). } \\
\text { Increased sky view factor. Reduced } \\
\text { albedo. } \\
\text { Snow cover }>10 \mathrm{~cm} \text { in depth. Low } \\
\text { admittance. High albedo. }\end{array}$ \\
\hline ind & $\begin{array}{l}\text { Low-rise and midrise industrial struc- } \\
\text { tures (towers, tanks, stacks). Few or } \\
\text { no trees. Land cover mostly paved } \\
\text { or hard-packed. Metal, steel, and } \\
\text { concrete construction materials. }\end{array}$ & d. dry ground & $\begin{array}{l}\text { Parched soil. Low admittance. Large } \\
\text { Bowen ratio. Increased albedo. } \\
\text { Waterlogged soil. High admittance. } \\
\text { Small Bowen ratio. Reduced albedo. }\end{array}$ \\
\hline
\end{tabular}

Figure 1. Local climate zone scheme [13].

Although LCZs are intended to facilitate objective measurements of UHI magnitude worldwide (i.e., as a difference in temperature between two LCZ types, e.g., $\left.\Delta \mathrm{T}_{\mathrm{LCZ1} / \mathrm{LCZD}}=\mathrm{T}_{\mathrm{LCZ1}}-\mathrm{T}_{\mathrm{LCZD}}\right)$, they also provide a useful discretization of the landscape with respect to its surface layer climate. Table 1 shows selected parameters of the LCZs demonstrating the broad spectrum of information on their physical properties that is delivered by the scheme. There are a number of reasons why the scheme has utility for compiling a worldwide urban morphological and urban metabolic database. First, there has been considerable uptake and general interest in this scheme within the field of urban climate. LCZs have 
been applied to case studies in many cities of the world and as such, there is a growing body of empirical evidence to support their use in urban climatology [16-19]. Given the disparity in how individual researchers interpret the urban landscape, LCZs provide a much needed context to standardize the classification of landscapes for ground-based climate studies. This results from the fact that the scheme was designed to be "sufficiently generic", i.e., it is inclusive of all regions and cultures and abandons excessive regional/architectural detail. Hence, the scheme has a reasonable level of complexity, while retaining universal meaning. Secondly, a full set of surface climatic properties is used to partition the landscape universe, meaning that the zones are differentiated by their structure, i.e., building and street dimensions, by the surface cover, such as the degree of permeability, the materials and the human activity or metabolism [13]. Finally, the scheme is well documented and easily definable vis-à-vis information sheets for every LCZ class. Each sheet specifies 10 quantifiable properties "relating to surface structure (sky view factor, aspect ratio, roughness element height), surface cover (plan fraction occupied by buildings, vegetation, and impervious ground), surface fabric (thermal admittance, surface albedo), and human activity (anthropogenic heat output)" [13], which have been gathered from a large body of literature and extensive field work. LCZ mapping therefore has the potential to yield valuable information on the basic physical properties of any urban area. However, since the scheme was not initially developed for mapping, a number of aspects require consideration with respect to its suitability for mapping purposes.

Table 1. Physical properties of the local climate zones, modified from [13], Tables 3 and 4. 1: Ratio of building plan area to total plan area (\%); 2: Ratio of impervious plan area (paved, rock) to total plan area (\%); 3: Mean height-to-width ratio of street canyons (LCZs 1-7), building spacing (LCZs 8-10), and tree spacing (LCZs A-G); 4: Ratio of the amount of sky hemisphere visible from ground level to that of an unobstructed hemisphere; 5: Geometric average of building heights (LCZs 1-10) and tree/plant heights (LCZs A-F) (m); 6: Ability of surface to accept or release heat $\left(\mathrm{J} \cdot \mathrm{m}^{-2} \cdot \mathrm{s}^{-1 / 2} \cdot \mathrm{K}^{-1}\right)$. Varies with soil wetness and material density; 7: Mean annual heat flux density $\left(\mathrm{W} \cdot \mathrm{m}^{-2}\right)$ from fuel combustion and human activity (transportation, space cooling/heating, industrial processing, human metabolism), varies significantly with latitude, season, and population density.

\begin{tabular}{cccccccc}
\hline $\begin{array}{c}\text { Local } \\
\text { Climate Zone }\end{array}$ & $\mathbf{1}\left(\boldsymbol{\lambda}_{\mathbf{b}}\right)$ & $\mathbf{2}\left(\boldsymbol{\lambda}_{\mathbf{I}}\right)$ & $\mathbf{3}\left(\boldsymbol{\lambda}_{\mathbf{S}}\right)$ & $\mathbf{4}\left(\boldsymbol{\psi}_{\text {sky }}\right)$ & $\mathbf{5}(\mathbf{m})$ & $\mathbf{6}\left(\mathbf{J} \cdot \mathbf{m}^{-\mathbf{2}} \cdot \mathbf{s}^{-1 / 2} \cdot \mathbf{K}^{-1}\right)$ & $\mathbf{7}\left(\mathbf{W} \cdot \mathbf{m}^{-\mathbf{2}}\right)$ \\
\hline LCZ 1 & $0.4-0.6$ & $40-60$ & $>2$ & $0.2-0.4$ & $>25$ & $1100-1200$ & $50-300$ \\
LCZ 2 & $0.4-0.7$ & $30-50$ & $0.75-2$ & $0.3-0.6$ & $10-25$ & $1000-2200$ & $<75$ \\
LCZ 3 & $0.4-0.7$ & $20-50$ & $0.75-1.5$ & $0.2-0.6$ & $3-10$ & $1000-2200$ & $<75$ \\
LCZ 4 & $0.2-0.4$ & $30-50$ & $0.75-1.25$ & $0.5-0.7$ & $>25$ & $1100-2000$ & $<50$ \\
LCZ 5 & $0.2-0.4$ & $10-50$ & $0.3-0.75$ & $0.5-0.8$ & $10-25$ & $1000-2200$ & $<25$ \\
LCZ 6 & $0.2-0.4$ & $20-50$ & $0.3-0.75$ & $0.6-0.9$ & $3-10$ & $1000-2200$ & $<25$ \\
LCZ 7 & $0.6-0.9$ & $<20$ & $1-2$ & $0.2-0.5$ & $2-4$ & $400-800$ & $<35$ \\
LCZ 8 & $0.3-0.5$ & $40-50$ & $0.1-0.3$ & $>0.7$ & $3-10$ & $1000-2000$ & $<50$ \\
LCZ 9 & $0.1-0.2$ & $<20$ & $0.1-0.25$ & $>0.8$ & $3-10$ & $1000-2200$ & $<10$ \\
LCZ 10 & $0.2-0.3$ & $20-40$ & $0.2-0.5$ & $0.6-0.9$ & $5-15$ & $1000-2500$ & $>300$ \\
\hline
\end{tabular}




\subsection{Is LCZ Mapping Appropriate?}

Some restrictions emerge from the origin of the LCZ scheme for characterizing screen-height air temperature observations. Identifying an ideal spot to place instruments in order to measure a representative local climate signal implies concentration on prototypes for a specific local climate (which means homogenous structures within a turbulent footprint) rather than mixed, indistinct or doubtful candidates. On the other hand, when discretising an entire city, each class will unavoidably have a certain amount of internal heterogeneity, since it is composed of a variety of urban landscapes that are part of a continuum rather than discrete types. This means that for classification purposes, the class boundaries, rather than centroids, become crucial, demanding a precise formulation of the boundaries between the classes. These considerations can be summarized in the following questions: Can an LCZ be assigned to any urban structure? Additionally, can only one LCZ be assigned to a given structure? Likewise, it can be asked, whether the classes are complete, comprehensive, and disjoint?

In general, no "overlaps" or "holes" exist for the parameter combinations of the 17 standard classes. However, it is possible to have outliers, but these are rare and are excluded from the standard set. For instance, sparse low-rise buildings on paved ground would be atypical of urban environments and do not warrant a class of their own. The scheme addresses these problems with the possibility to define subclasses, which are essentially mixtures between the standard classes (in this case LCZ 9E is used to define sparse low-rise buildings on paved ground). Hence, the scheme does not only consider the full spectrum of possible combinations but also a priori knowledge about the frequency of their appearance. This takes advantage of the empirical observation that certain parameter values tend to cluster in cities - Compact high-rise buildings are, for instance, usually associated (spatially) with extensive paved ground, few trees, low albedo and large anthropogenic heat flux. This is underpinned by experience and discussions with experts from different urban areas and cultures, which confirm that LCZs can be applied in many parts of the world.

Nevertheless, the heterogeneity of urban structures means that some areas can be considered as unclear or fuzzy in terms of LCZ membership. For instance, the chosen threshold of $10 \mathrm{~m}$ height between low-rise and mid-rise LCZ classes only reflects the mean height and not the variance, which can make the classification of non-uniform ensembles difficult. Here, some contextual expert knowledge is needed since in the case of a mixture of $8 \mathrm{~m}$ and $12 \mathrm{~m}$ buildings, the difference in terms of their climatological bearing is so small that either a low or mid-rise class might be acceptable. Conversely, if the separation between building heights becomes much greater (e.g., $30 \mathrm{~m}$ buildings mixed with $3 \mathrm{~m}$ buildings), this certainly affects the roughness characteristics and hence the assignment of subclass becomes necessary (LCZ $1_{3}$ to denote compact high-rise mixed with compact lowrise). Again, due to spatial clustering of surface properties in cities, this combination is rare. Similarly, structures with extreme cover fractions might not match any of the predefined classes.

Overall, we believe the existing LCZ classes provide a disjoint and largely complementary discretisation of the (urban) landscape universe that covers the vast majority of existing urban forms. They are well balanced between accuracy and universality. The definition of subclasses should be allowed in specific instances where the climatic effect is not negligible and the subclass is applicable to larger areas, while the misclassification of single pixels should be acceptable. Generally, subclasses should be used cautiously since the higher accuracy comes at the cost of reduced universality. This trade-off is, 
however, not specific to LCZ mapping but a general problem in pattern recognition where a potentially unlimited variety of signals are reduced to a discrete number of patterns. For this reason, the protocol should include a mechanism to document and report instances of urban structures that are currently not sufficiently covered within the standard set of LCZs.

Having accepted that LCZs are suitable for mapping urban areas, the next logical step is to identify an appropriate mapping method suitable for global application.

\subsection{A Suitable Method for LCZ Mapping}

The suitability of a LCZ mapping method depends on the application. As stated before, due to the culturally neutral nature of the LCZ scheme, the individual classes will inherently have different spectral properties in different parts of the world, which makes local training data and knowledge of the local urban structures a necessity. Based on this, we aim to derive a simple workflow that is established in a protocol to enable local operators from all parts of the world with different backgrounds (likely without remote sensing training and experience) to derive a LCZ map as a basic representation of the physical properties of their city for WUDAPT. This implies a number of restrictions. Firstly, the procedure should be universal and have a low level of data requirements, i.e., not be reliant on specific/detailed datasets such as detailed building morphological data or 3D models; these are only available for a few cities. Secondly, it should be as objective as possible meaning that the results should be comparable for different cities and operators. Thirdly, it should be computationally and fiscally inexpensive. More specifically, the processing on a single core should take less than 10 minutes and it should be based on free and globally available data and software. To maintain comparability, auxiliary data should be used for validation or later refinement rather than for the initial classification. Furthermore, it should be intuitive and easy to use meaning that the procedure can be completed in one day without extensive prior knowledge.

Several methods to derive LCZs have been proposed and applied for LCZ mapping, including a manual sampling of individual grid cells using Geo-Wiki, digitisation of homogenous LCZs, a GIS-based approach using building data [20], object-based image analysis [5,21] and supervised pixel-based classification [14]. Methods to derive other "urban structural types" [22-24] are not considered here, since the classification schemes are usually designed for a specific purpose and cultural/regional background and therefore do not meet the universality requirement. No single method can entirely fulfil the aforementioned criteria. Both the manual sampling of grid cells and the digitisation of the entire urban area require substantial expert knowledge and results have shown large differences between operators. Moreover, the manual sampling of grid cells is very time-consuming.

The GIS-based approach [20] was tested for Szeged, Hungary and is conducted on lot area polygons (building blocks and the adjacent area) derived from a 3D building database. For each city block, morphological and radiative parameters are extracted from the same database and high resolution RapidEye satellite imagery, which are subsequently aggregated to patches of sufficient size. Therefore, two of the best fitting LCZ categories are assigned to every polygon and a posterior rule-based filtering is used to generate larger homogenous patches. The approach is quite objective, but requires specific data that are generally unavailable for most cities. However, the rule-based post-classification filtering is potentially a good alternative for post-processing for WUDAPT. 
The object-based image analysis approach [5,21] was tested in two case studies for Xuzhou, China and Atlanta, US. It is based on two processing chains, first delimiting blocks and secondly using spatial and spectral indices to assign LCZs to the extracted polygons. The results are promising, but the method depends on high resolution data, e.g., from the Japanese Panchromatic Remote-sensing Instrument for Stereo Mapping (PRISM) on the Advanced Land Observing Satellite, which is usually not freely available. In addition, the transferability of the method still needs to be investigated. A substantial disadvantage in using this methodology for the community-based approach in WUDAPT is its requirement for substantial image processing expertise, which makes the method too complex for an inexperienced user.

The supervised pixel-based approach showed good results with overall classification accuracies of up to $97 \%$ for Hamburg, Germany [14]. This method is assumed to be comparably robust and, although dependent on digitised training data, is largely objective and thus generally well suited for the purpose. However, the case study employed different classifiers including multi-layer perceptron neural networks, support vector machines and random forest software from a java library, which is too difficult for inexperienced users to operate, and MATLAB, which is proprietary software. Furthermore, a large number of input features were derived from multi-temporal Landsat thermal and multispectral data as well as a digital (building and tree) height model from interferometric airborne SAR, which are difficult to compute and not widely or freely available. To achieve the aims of universality while maintaining a straightforward process, the workflow was modified and considerably simplified (see Section 3/Figure 5). Among the previously tested classifiers, random forest [25] was chosen as an ideal compromise between the achieved accuracy and computational performance. Moreover, the classifier has a few additional advantages. First, it is non-parametric, which is essential since each LCZ can have quite different instances or appearances within one city. Further, random forest provides an unbiased error estimate without requiring additional testing data.

\subsection{Suitable Features for LCZ Mapping}

To conduct an automated classification of LCZs, discriminative features are needed. The characteristics provided in the LCZ information sheets are not ideal for a supervised classification for a number of reasons. Since they were designed to provide broad information about observational climate sites, there is substantial redundancy and overlap in single LCZ characteristics. Figure 2 provides a representation of LCZs by selected characteristics, namely sky view factor (SVF) and the percentage of impervious surface area (\%ISA) (left), as well as height (representing the urban structure) and building fraction (representing urban cover) (right). This demonstrates that several features are needed while not all features are equally important for discrimination. Furthermore, not all features can be observed directly by Earth observations. Thus, it requires us to determine which observable spectral characteristics can be used to substitute them.

As stated previously, the LCZs are differentiated according to the morphology of streets and buildings (urban structure), surface permeability (urban cover), construction materials (urban fabric) and human activity (metabolism, i.e., release of anthropogenic heat). Unfortunately, not all of these surface climatic properties can be observed by remote sensing methods in a straightforward manner. Urban metabolism is difficult to observe from space (nightlight and chemical species in coarse resolution might be considered as exceptions). Urban structure can only be analysed with very high resolution data, which 
are usually not free of charge and require advanced processing as well as the incorporation of complex contextual knowledge. However, materials and their morphology can readily be observed using moderate resolution multispectral satellite imagery. Indirect measurements such as permeability can be derived from other indicators such as NDVI (normalized difference vegetation index).

a)

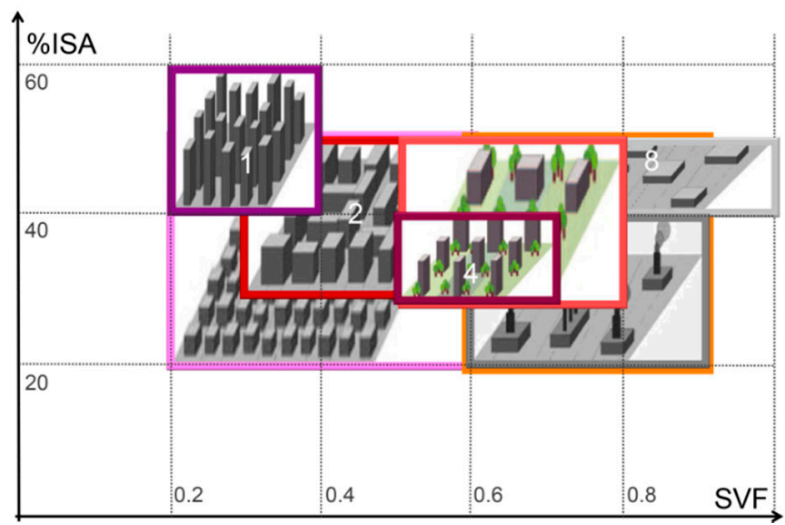

b)

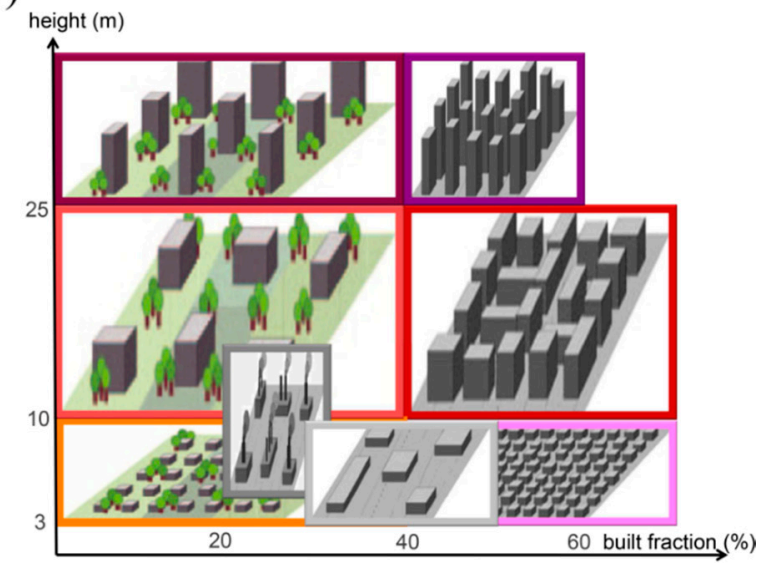

Figure 2. Potential separation of built LCZ classes according to selected feature pairs. (a) Sky view factor (SVF) and percentage of impervious surface area (\%ISA); (b) built fraction and building height.

In Figure 3, the LCZ scheme was sorted in a simplified manner along two axes representing height as the most important characteristic of urban structure and permeability as the most important characteristic of the urban fabric and cover. The arrows indicate potential wavelength bands and features for discrimination. While bare soil and sand can be well distinguished in the near and shortwave infrared, the compact and open LCZ can best be distinguished by their vegetation fraction using NDVI or fractional cover. Furthermore, the imperviousness is well correlated with land surface temperature [26] and hence the thermal infrared wavelength band can be applied here for discrimination.

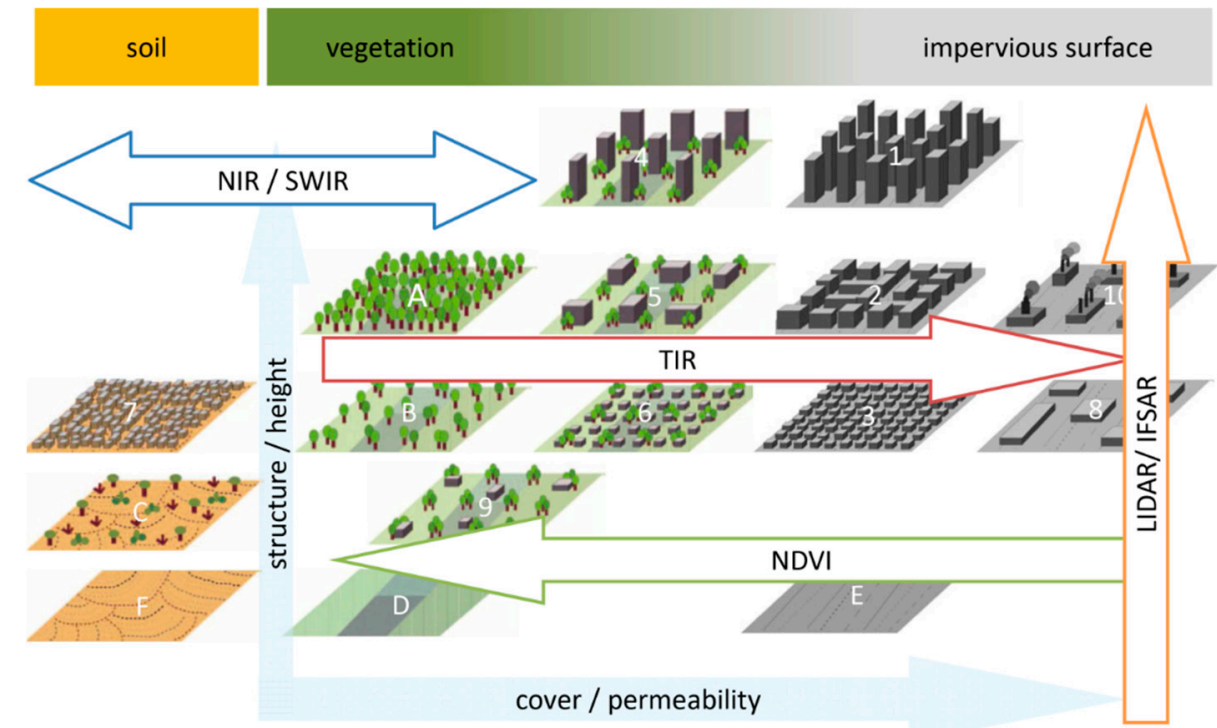

Figure 3. Observable characteristics to distinguish LCZs from remote sensing data. 
To identify urban structure, Landsat imagery offers the largest archive of moderate resolution multi-spectral data spanning more than 40 years, and is available free of charge from the USGS [27]. Height data are more difficult to obtain. Existing global datasets from the Shuttle Radar Topography Mission (SRTM) and the ASTER Global Digital Elevation Map only provide a surface model and are too coarse and noisy to extract urban structures. The TanDEM-X radar constellation will provide a global dataset in 0.4 arcsec resolution in the future but this is not yet available and will be subject to proprietary copyright. The urban structure must therefore be differentiated based on spectral differences in the material and cover.

\subsection{The Matter of Scale}

Different aspects must be reflected when identifying the optimal or most appropriate scale for a pixel-based classification of LCZs. Most importantly, three aspects need consideration. First, the concept of LCZs itself poses certain limitations. They are defined as "hundreds of meters to several kilometers in horizontal scale" [13]; this is sometimes referred to as a local (or neighbourhood scale) and lies between climatological micro- and meso-scales. This concept allows a certain range of appropriate scales, which implies different "valid" LCZ maps depending on the resolution. Figure 4 shows this scale dependency with possible classification results in $1000 \mathrm{~m}$ resolution (left) and $200 \mathrm{~m}$ (right) for Phoenix Park, Dublin. While both could be deemed as appropriate definitions of the local scale in terms of the overall LCZ concept, the finer resolution results in a much larger fragmentation of the classification as would be expected.

Secondly, pattern recognition restrictions have to be considered for the optimal scale. Since urban areas are composed of a large number of objects, at the classification scale, each pixel must consist of a comparable fraction of the characteristic surface covers for a specific urban type (i.e., roofs, street, grass, trees, cars, etc.). This means that the sampling distance should be larger than the characteristic length of the neighbourhood, which, for instance, could be defined by the distance between two buildings or the size of a building block. If it becomes smaller than that, the individual pixel will classify single surface covers rather than local scale structures resulting in very noisy output. Conversely, if the sampling distance is smaller than the average size of the homogenous urban areas (i.e., the LCZs) needed to minimise the percentage of mixed pixels, there has to be a sufficient number of large samples of homogenous pixels to preserve representative spectral information. Unfortunately, the characteristic length and accordingly the best pixel size will likely differ between urban structures and also between cities. Likewise, the extent of homogenous urban structures may vary between cities of different size and location. Hence, there may not be one "optimal" scale for LCZ classification. However, an approximate range can be specified. A resolution of $10-30 \mathrm{~m}$ is too high; equally a resolution of 500-1000 $\mathrm{m}$ is too low. Preliminary tests for different cities showed 100-150 $\mathrm{m}$ to be a good compromise.

Third, user requirements need to be considered. Different models are used to simulate urban effects on various scales from the building block scale [28]; meso scale [29] up to global models [30]. Potential use cases should therefore be evaluated and considered before a final protocol is established.

Since the needed scales will likely span several orders of magnitude and not all use cases are previously known, an aggregation scheme should be part of the protocol and portal tool. This is far from trivial, because LCZs are only defined for a specific range of scales and the homogeneity criterion will unavoidably be violated if they are aggregated to coarser scales. Therefore, it is appropriate to conduct the 
classification on a finer scale and aggregate the extracted urban canopy parameters rather than the LCZ to the coarser scale, which would preserve some of the sub-grid scale heterogeneity for model applications.

Because zones are not arranged on a regular grid, the optimal resolution for a pixel-based classification (e.g., $100 \mathrm{~m}$ ) should be systematically higher than the preferred LCZ scale (hundreds of meters to kilometres). For example, while a large garden or small park of 1 hectare might fill an entire pixel, in isolation it does not constitute a LCZ. A simple way to (a) decrease the granularity, (b) erase small discontinuous areas, and c) bring a limited amount of contextual knowledge to the mapped product is to apply post classification filters (e.g., majority, clamp and sieve or morphological filters). Thus, the question of granularity can be reduced to the question: how large should the smallest preserved patch be? Moreover, the shape of the patches could also be controlled to a certain extent, since the neighbourhoods are not necessarily rectangular.
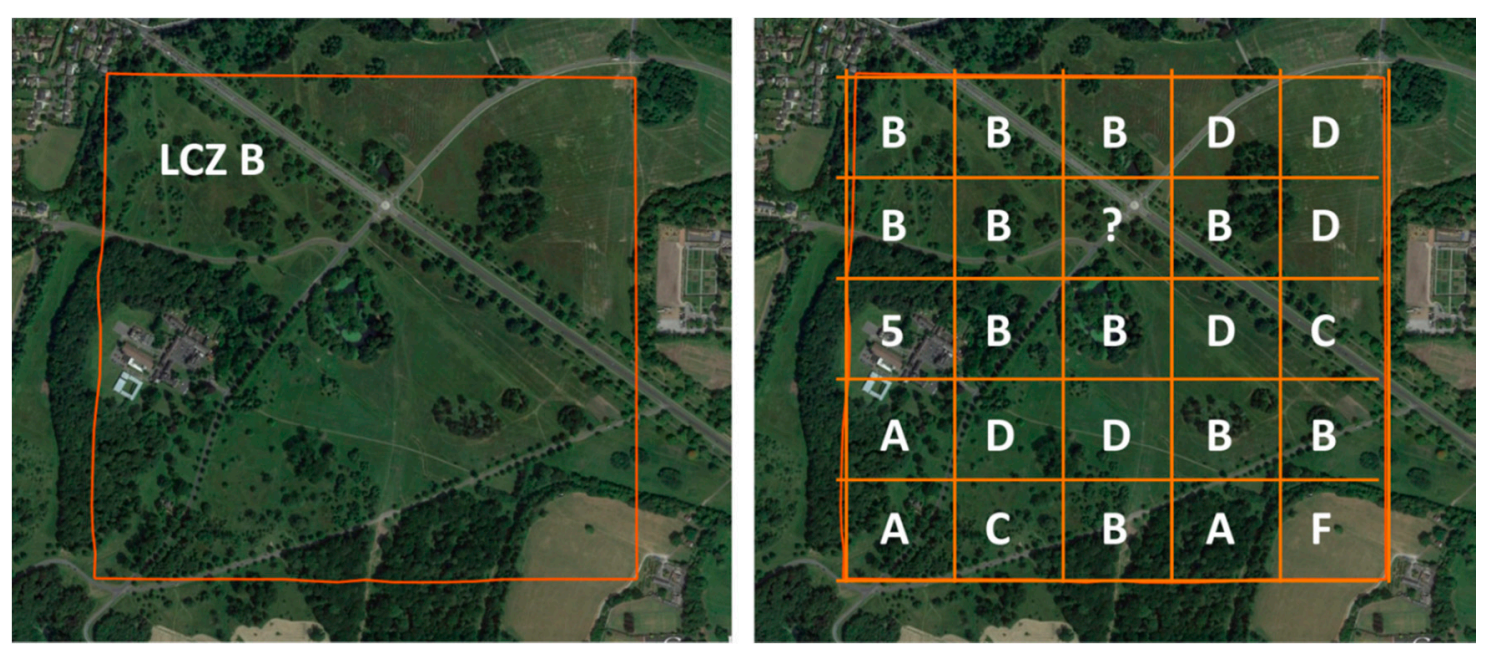

Figure 4. Scale dependency of the LCZ concept. Possible classification results in $1000 \mathrm{~m}$ resolution (left) and $200 \mathrm{~m}$ (right) for Phoenix Park, Dublin. Imagery: Google Earth.

\section{Implementation and Proof of Concept}

Important aspects and tools of the implemented workflow are shown in Figure 5. As stated before, the intended mapping methodology shall be universal, simple and objective. Furthermore, it shall be fast, free of proprietary data or software and simple enough to allow local operators without remote sensing training to conduct and validate LCZ-classifications for their cities, since local expert knowledge is required. This is incorporated by local users identifying appropriate locations that can be used to train the LCZ classification scheme for their specific city. We decided to use Google Earth, which is free (even if proprietary), easy to use, and includes very high resolution imagery for most cities (Figure $5 b$ ) for assisting users to identify appropriate training sites. The Landsat data can easily be acquired from the U.S. Geological Survey Earth explorer interface (Figure 5a), and has a resolution of $30 \mathrm{~m}$. Since December 2014, a provisional Landsat 8 surface reflectance product is available which is preferred in order to reduce atmospheric influences on the classification. However, generally top of the atmosphere radiance is sufficient for our purposes [31]. Instead of explicitly computing NDVI and land surface temperature, for simplicity the red, near infrared and thermal bands were considered directly, which had negligible influence on the classification accuracy. Since the recent (post 2003) Landsat 7 scenes are 
affected by a scan line correction error, Landsat 8 data [32] are recommended. Depending upon availability, scenes from different seasons should be selected to include phenological information in the classification. For the geometrical preprocessing and the classification we have chosen the System for Automated Geoscientific Analyses (SAGA, [33]) as a platform. SAGA-GIS has been designed for an easy and effective implementation of spatial algorithms and, hence, serves as a framework for the development and implementation of geoscientific methods and models. It provides an easily approachable user interface with many visualization options (Figure 5c) and can be scripted from the command line, using python or R. In version 2.1.4, SAGA provides more than 700 methods including file operations, referencing and projection, basic spatial raster and vector operations, and filters, which are used here in the pre- and post-processing phases. Since the random forest classifier was not available in SAGA before, it was implemented from the image processing library VIGRA [34]. The latest SAGA version supports tool-chains, allowing the subsequent call of several modules by a single click, which will help to further simply the classification procedure.

a)

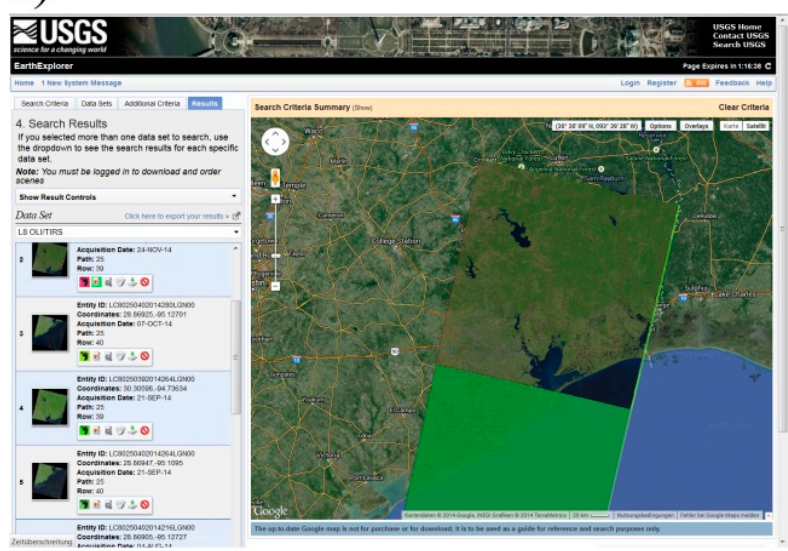

c)

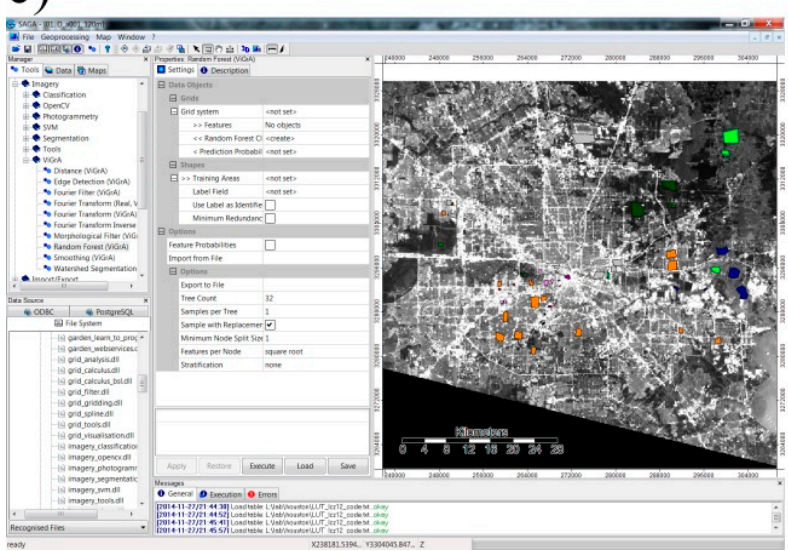

b)

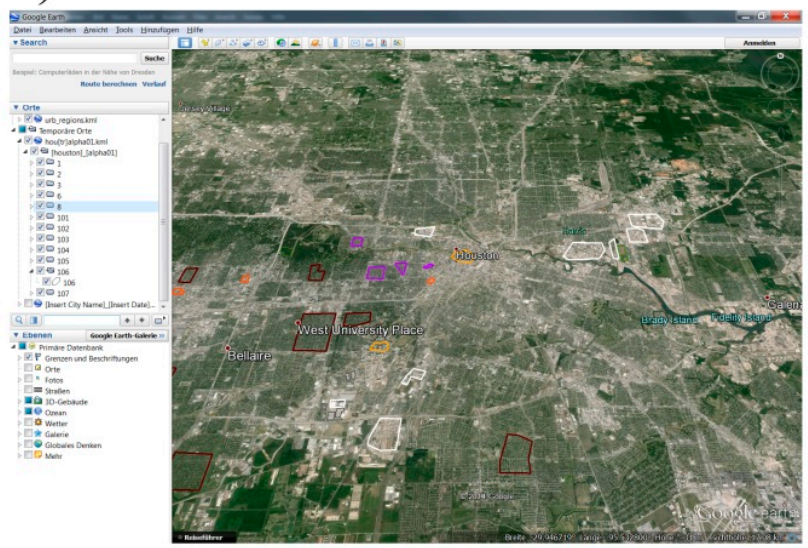

d)

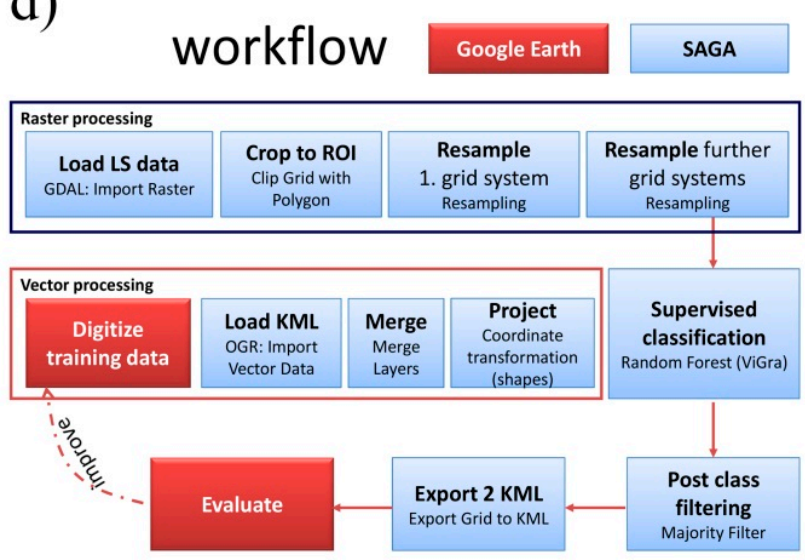

Figure 5. Overview of the mapping procedure. (a) Acquisition of Landsat data in USGS Earth-Explorer; (b) digitization of training areas in Google Earth; (c) classification in SAGA-GIS; (d) workflow.

We also considered Google Earth Engine [35] as a classification platform. This platform provides a powerful system for online processing and visualisation of large geospatial datasets on Google servers, which has advantages like online data availability and management. However, Google Earth Engine is 
currently available for trusted testers only and we preferred to develop the methodology for WUDAPT on an independent platform. Nevertheless, the collection of training data in Google Earth in principle allows later processing in Google Earth Engine.

To test the workflow the urban areas of Hamburg, Germany, Dublin, Ireland, and Houston, USA were classified. Results are presented for visual interpretation in Figure 6. In general, the urban structures in terms of actual LCZ types of all cities are well represented and accurately delineated. The target grid size varied between $100 \mathrm{~m}$ and $120 \mathrm{~m}$, as both were deemed suitable in relation to the pattern recognition problem discussed previously. In terms of the LCZ concept, however, the resulting patches were too fragmented and hence post-classification filtering was considered necessary.

a)

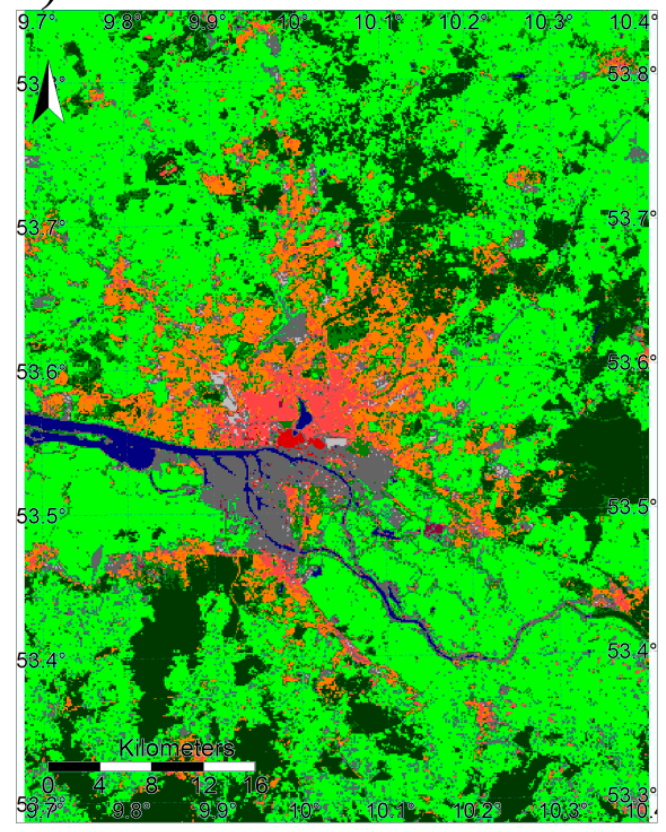

c)

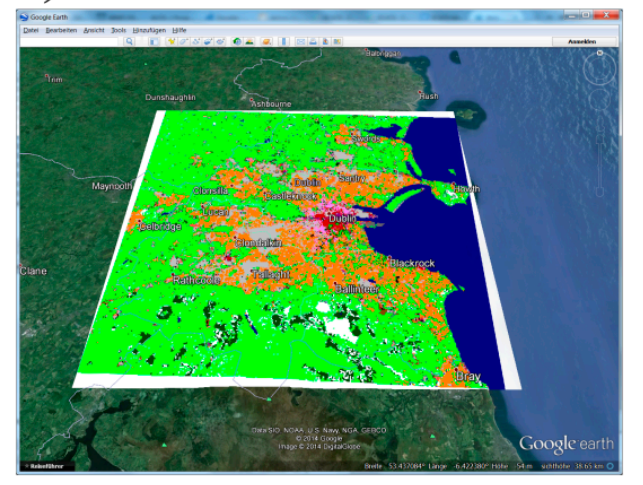

b)

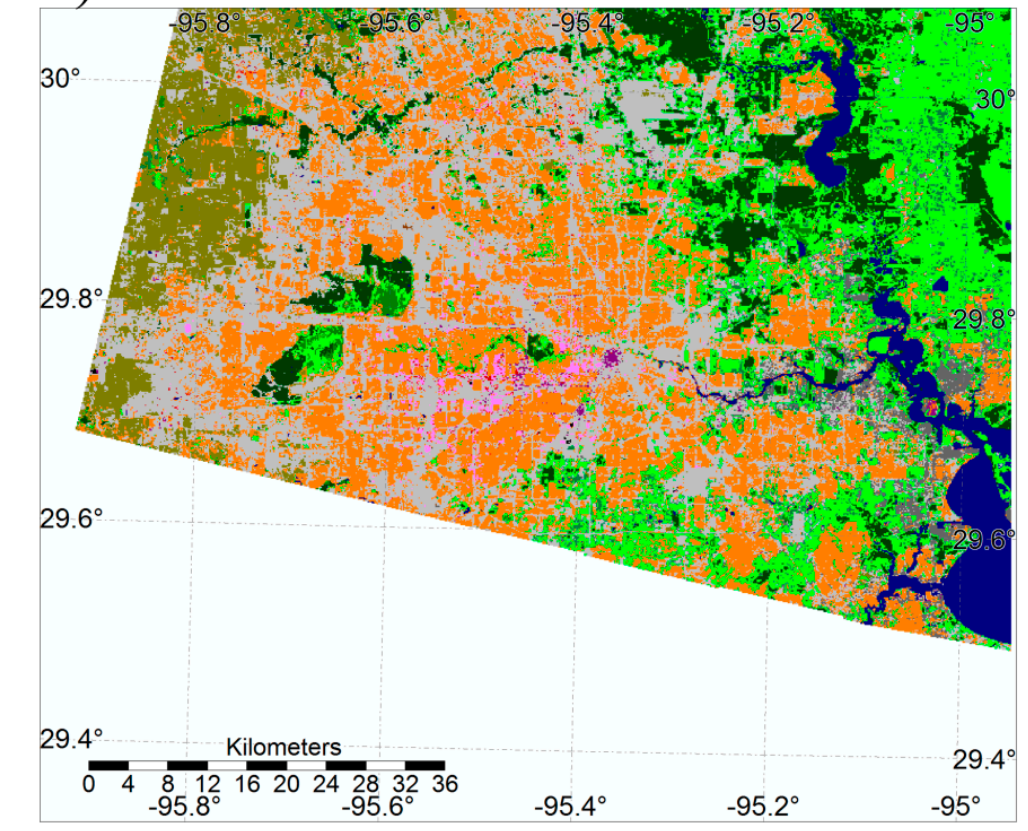

d)

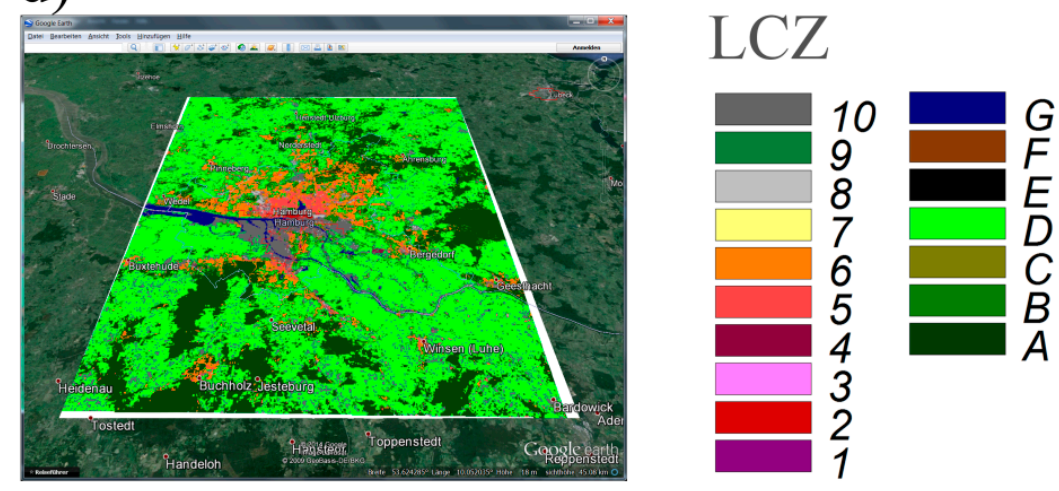

Figure 6. Local Climate Zones (LCZ) classification results for (a) Hamburg, Germany; (b) Houston, USA; (c) Dublin, Ireland in Google Earth; (d) Hamburg in Google Earth. 
Table 2. Mean and standard deviation of out of bag error from five classifications in $\%$ and sum of computing time in minutes for Houston using different numbers of trees and feature sets. Feature sets: LT50250392010077EDC00 and LC80250392013101LGN01 refer to a single Landsat (LS) 5 and LS 8 scene, respectively, same without TIR: LS8 without thermal infrared bands, all LS8: five LS8 scenes, all TIR: thermal bands from LS8LS5, LS8LS5: LS8 plus six LS5 scenes.

\begin{tabular}{|c|c|c|c|c|c|c|}
\hline Number of Trees & 4 & 8 & 16 & 32 & 64 & 128 \\
\hline \multicolumn{7}{|l|}{ Mean out of bag error $/ \%$} \\
\hline LT50250392010077EDC00 & 15.3 & 14.6 & 13.9 & 13.6 & 13.4 & 13.4 \\
\hline LC80250392013101LGN01 & 14.0 & 13.4 & 12.5 & 12.3 & 12.1 & 12.0 \\
\hline same without TIR & 15.9 & 15.0 & 14.3 & 13.7 & 13.5 & 13.4 \\
\hline all LS8 ${ }^{1}$ & 12.8 & 12.3 & 11.7 & 11.3 & 11.3 & 11.2 \\
\hline all TIR & 14.9 & 13.9 & 13.0 & 12.5 & 12.2 & 12.2 \\
\hline $\mathrm{LS}_{8} \mathrm{LS}^{2}$ & 12.6 & 12.1 & 11.5 & 11.1 & 11.0 & 11.0 \\
\hline \multicolumn{7}{|l|}{ Standard deviation/\% } \\
\hline LT50250392010077EDC00 & 0.35 & 0.21 & 0.19 & 0.12 & 0.13 & 0.06 \\
\hline LC80250392013101LGN01 & 0.18 & 0.20 & 0.08 & 0.11 & 0.08 & 0.05 \\
\hline same without TIR & 0.13 & 0.10 & 0.19 & 0.10 & 0.20 & 0.14 \\
\hline all LS8 ${ }^{1}$ & 0.26 & 0.11 & 0.08 & 0.08 & 0.13 & 0.06 \\
\hline all TIR & 0.33 & 0.24 & 0.14 & 0.18 & 0.09 & 0.07 \\
\hline LS8LS5 $^{2}$ & 0.21 & 0.09 & 0.10 & 0.10 & 0.09 & 0.10 \\
\hline \multicolumn{7}{|l|}{ Total computing time/min } \\
\hline LT50250392010077EDC00 & 0.17 & 0.21 & 0.24 & 0.43 & 0.70 & 1.22 \\
\hline LC80250392013101LGN01 & 0.18 & 0.21 & 0.26 & 0.38 & 0.66 & 1.16 \\
\hline same without TIR & 0.16 & 0.19 & 0.26 & 0.36 & 0.60 & 1.10 \\
\hline all LS8 ${ }^{1}$ & 0.44 & 0.57 & 0.82 & 1.31 & 2.19 & 4.01 \\
\hline all TIR & 0.22 & 0.26 & 0.34 & 0.51 & 0.89 & 1.83 \\
\hline LS8LS5 $^{2}$ & 0.75 & 0.94 & 1.34 & 2.12 & 3.65 & 6.57 \\
\hline
\end{tabular}

${ }^{1}$ LC80250392013101LGN01, LC80250392013133LGN01, LC80250392013229LGN00, LC80250392013293LGN00, LC80250392013357LGN00; ${ }^{2}$ LS8 and LT50250392010077EDC00, LT50250392010125EDC00, LT50250392010237EDC00, LT50250392011240EDC00, LT50250392011256EDC00, LT50250392011304EDC00.

Table 2 presents a quantitative analysis of the out of bag error using different feature sets and number of decision trees for Houston, USA. Since the results vary slightly due to the random nature of the classifier, each classification was conducted five times; the mean and standard deviation of the five attempts are presented. While the single Landsat 8 scene performed better than the single Landsat 5 scene (likely due to the higher number of bands and radiometric accuracy), both were clearly outperformed by multi-temporal sets of scenes from different dates. A set of five Landsat 8 scenes showed very promising results $(11.2+/-0.06 \%$ with 128 trees $)$ while six additional Landsat 5 scenes only marginally improved this result further $(11.0+/-0.10 \%$ with 128 trees). The combination of thermal and multi-spectral data also seems essential. A larger number of decision trees generally resulted in a higher accuracy and lower variation of the error (e.g., $15.3+/-0.35 \%$ for the LS5 with four trees and $13.4+/-0.06 \%$ with 128 trees). Both the number of features and number of trees considerably influence the computing time. However, even the largest set with 128 trees was processed in less than 
seven minutes using a standard personal laptop with four cores with $2.1 \mathrm{GHz}$. This more than satisfies the computational time criterion, particularly since the low standard derivation suggests that multiple runs are not necessary. From this analysis, it appears a large number of trees should be employed for generating the final LCZ classification for a particular city, while a smaller number are sufficient for testing purposes. A more comprehensive investigation of the optimal classification scale, training samples and input parameters are currently being conducted on 16 cities worldwide which were included in an initial international expert workshop for WUDAPT held in Dublin in 2014, and will be presented in a subsequent paper.

Table 3 shows the confusion matrix for the classification of Houston using the Landsat 8 data and 128 trees. These values are based on the training data and, therefore, the error estimates are not a reliable estimate of the overall accuracy. As they draw from the training data, the resulting error estimates are routinely lower than the out of bag error, which is an independent error estimate (overall accuracy $=0.96$, out of bag error $=0.11$ ). Nevertheless, the confusion matrix is useful for examining the results arising from individual LCZ classes. A certain amount of misclassification occurred between the urban classes (LCZs 2, 3, 6 and 8), which is to be expected given the continuum of structures which exist in reality. Likewise, similar spectral signatures lead to misclassification between paved surfaces (LCZ E) and large low-rise (LCZ 8) and, consequently, a low producer accuracy of 0.47 for LCZ E.

Table 3. Confusion Matrix for the classification results of Houston using 128 trees and five Landsat 8 scenes. Besides the overall accuracy (OA), the user accuracy (UA), producer accuracy (PA), and $\kappa$ coefficient are provided. Evaluation is based on training data only. Rows refer to classification output, columns to reference data.

\begin{tabular}{ccccccccccccccccc}
\hline $\mathbf{L C Z}$ & $\mathbf{1}$ & $\mathbf{2}$ & $\mathbf{3}$ & $\mathbf{6}$ & $\mathbf{8}$ & $\mathbf{9}$ & $\mathbf{1 0}$ & $\mathbf{A}$ & $\mathbf{B}$ & $\mathbf{C}$ & $\mathbf{D}$ & $\mathbf{E}$ & $\mathbf{F}$ & $\mathbf{G}$ & $\boldsymbol{\Sigma}$ & $\boldsymbol{U A}$ \\
\hline 1 & 78 & 0 & 2 & 0 & 3 & 0 & 0 & 0 & 0 & 0 & 0 & 0 & 0 & 0 & 83 & 0.94 \\
2 & 0 & 24 & 1 & 0 & 1 & 0 & 0 & 0 & 0 & 0 & 0 & 0 & 0 & 0 & 26 & 0.92 \\
3 & 0 & 3 & 140 & 4 & 2 & 0 & 0 & 0 & 0 & 0 & 0 & 2 & 0 & 0 & 151 & 0.93 \\
6 & 0 & 1 & 10 & 1470 & 15 & 0 & 0 & 0 & 0 & 1 & 1 & 2 & 0 & 7 & 1507 & 0.98 \\
8 & 12 & 15 & 12 & 19 & 1364 & 0 & 9 & 0 & 0 & 4 & 0 & 21 & 7 & 0 & 1463 & 0.93 \\
9 & 0 & 0 & 0 & 1 & 0 & 24 & 0 & 0 & 0 & 0 & 4 & 0 & 0 & 0 & 29 & 0.83 \\
10 & 0 & 0 & 0 & 1 & 2 & 0 & 77 & 0 & 0 & 0 & 0 & 0 & 0 & 1 & 81 & 0.95 \\
$\mathrm{~A}$ & 0 & 0 & 0 & 0 & 0 & 0 & 0 & 677 & 0 & 0 & 0 & 0 & 0 & 1 & 678 & 1.00 \\
$\mathrm{~B}$ & 0 & 0 & 0 & 0 & 0 & 0 & 0 & 1 & 100 & 0 & 0 & 0 & 0 & 3 & 104 & 0.96 \\
$\mathrm{C}$ & 0 & 0 & 0 & 0 & 0 & 0 & 0 & 0 & 0 & 209 & 0 & 0 & 0 & 0 & 209 & 1.00 \\
$\mathrm{D}$ & 0 & 0 & 0 & 17 & 4 & 11 & 0 & 10 & 9 & 0 & 692 & 0 & 0 & 4 & 747 & 0.93 \\
$\mathrm{E}$ & 0 & 0 & 1 & 0 & 0 & 0 & 0 & 0 & 0 & 0 & 0 & 22 & 0 & 0 & 23 & 0.96 \\
$\mathrm{~F}$ & 0 & 0 & 0 & 0 & 0 & 0 & 0 & 0 & 0 & 0 & 0 & 0 & 11 & 0 & 11 & 1.00 \\
$\mathrm{G}$ & 0 & 0 & 0 & 0 & 0 & 0 & 0 & 0 & 0 & 0 & 0 & 0 & 0 & 1042 & 1042 & 1.00 \\
$\Sigma$ & 90 & 43 & 166 & 1512 & 1391 & 35 & 86 & 688 & 109 & 214 & 697 & 47 & 18 & 1058 & $\kappa$ & 0.96 \\
$P A$ & 0.87 & 0.56 & 0.84 & 0.97 & 0.98 & 0.69 & 0.90 & 0.98 & 0.92 & 0.98 & 0.99 & 0.47 & 0.61 & 0.98 & $0 A$ & 0.96 \\
\hline
\end{tabular}

\section{Conclusions and Outlook}

In this paper, we have presented conceptual considerations regarding a standard protocol to derive LCZs from remote sensing data. The overall aim is the design of a universal, simple and objective mapping method that allows local experts without GIS and remote sensing experience to conduct and 
validate LCZ-classifications for their respective cities. Beyond its original purpose to classify UHI observation sites, the LCZ scheme was found to provide a generic and largely comprehensive and disjoint discretisation of the urban landscape with respect to its canopy layer climate. The scheme is well balanced between accuracy and universal applicability. Therefore, we considered it as an optimal starting point for the collection of more comprehensive data on the physical and, in particular, climatologic characteristics of urban areas. To address regional and cultural idiosyncrasies, subclasses can be used but this should be limited to larger areas of uniform specific structures. The use of these additional classes should be well documented and collected in order to standardize it for cultural and biophysically similar regions.

Different mapping methods were discussed and a pixel-based supervised classification based on multi-temporal Landsat data was found to be the best compromise regarding the given requirements (universality, objectivity, computing and monetary cost, and limited training of the operators). A random forest classifier was implemented in the open source GIS SAGA software, which provides an easy to use graphical user interface for post-processing and classification. Initial case studies have been conducted as a proof of concept with promising results. Our findings suggest that spectral information from remote sensing data was commonly sufficient for supervised LCZ classification in moderate humid climates; classification of cities in other climate regimes is currently being tested. Multi-temporal data representing seasonal phenological stages seem to improve the classification accuracy although the optimal seasonal distribution of images needs further investigation and may vary by climate. Furthermore, the added value of data from other sensors and auxiliary sources such as OpenStreetMap will be investigated. However, it has been emphasized that global availability and universality is an important requirement in the context of this project.

Optimal scale should be considered from three different perspectives: the LCZ concept itself, pattern recognition restrictions and user demands. Since the layout of the LCZ is not a regular grid, the second aspect necessarily demands a resolution that is finer than the granularity of the concept. Therefore, the initial classification should be conducted at a resolution coarser than individual buildings and smaller than the homogenous structures. The optimal scale might vary between classes and cities, but we consider $100 \mathrm{~m}$ as a default value. The initial classification should be filtered to derive homogenous structures in accordance with the LCZ concept by post-classification filters. This is currently preferred over more sophisticated aggregation methods like reclassification kernels [36] or object-based image analysis [37] due to the community-based approach. However, the performance of this approach will be evaluated against these other methods in the future. From additional tests in different cities, there is some evidence that existing urban morphologies (especially in historic towns of medium size) might be more heterogeneous than the LCZ scheme can accommodate. Furthermore, an aggregation scheme to derive user data at coarser resolutions should be part of the protocol. Therefore, potential use cases should be evaluated and considered before a final protocol is established. The final protocol will contribute to a larger initiative aiming at the compilation of worldwide information on urban structure, materials and metabolism in a framework called WUDAPT $[3,11]$ and eventually lead to a physical census of cities.

In WUDAPT, data collection will be conducted in different stages, each representing different levels of detail. The described methodology is intended to spatially classify cities into the LCZ scheme at a level 0 data stage, laying the foundation for further development. Higher level products will contain more information about various city and area specific parameters describing aspects of urban form (that 
is, structure, e.g., building height), cover (e.g., vegetated area fraction), materials (e.g., roof type), and function (e.g., building air conditioning and heating). LCZ mapping (level 0) will decompose cities into distinct urban landscapes based on a range of physical parameters (Table 1) that generate similar thermal signatures at neighborhood-scale [12]. Since the LCZs have associated generic ranges of parameters (Table 1), urban climate models can be used to provide a first assessment of the climatic properties of the city. The methodology described here is solely intended to provide a universal, simple and objective mapping methodology. We expect that additional details can be added at later stages of the WUDAPT process to allow supplementary information about building materials, building form and building use to further differentiate LCZ related climate impacts. The initial implementation of WUDAPT will be conducted with contemporaneous Landsat data inputs. However, in principle, historical as well as future Landsat data can be employed to provide historical as well as updated descriptions of LCZs given sufficient training data. If historic data are required, intersection with the global human settlement layer can also be considered. When fully implemented, WUDAPT will provide a powerful resource for performing community-based urban model applications for a variety of weather, climate and air quality problems, ultimately superseding initial related efforts such as NUDAPT [38], which provided urban canopy parameters for 44 USA cities.

\section{Acknowledgments}

We thank the USGS and the NASA for the free Landsat data, the SAGA user group and developers for the free GIS software, Google for Google Earth, and the American Meteorological Society (AMS) for the permission to use the materials describing the LCZ (especially Figure 1). Further, we thank the participants of the WUDAPT Workshop 2014 in Dublin for their valuable comments on the methodology and the reviewers for their helpful and fruitful remarks on the first manuscript. Finally, we would like to acknowledge the organizations, initiatives and projects that provided support for the workshop including University College Dublin, Argonne National Laboratory, the FP7 ERC grant CrowdLand (No. 612755) and COST Action TD1202.

\section{Author Contributions}

Olaf Conrad and Jürgen Böhner were responsible for implementation and testing of the workflow in SAGA. All other authors equally contributed to the idea of the paper and the discussion of its content at the workshop in Dublin. The first draft was mainly written by Benjamin Bechtel and the manuscript was intensively revised several times by all authors. The case studies were conducted by Benjamin Bechtel and Paul Alexander.

\section{Conflicts of Interest}

The authors declare no conflict of interest.

\section{References}

1. Mills, G. Cities as agents of global change. Int. J. Climatol. 2007, 27, 1849-1857.

2. Oke, T.R. City size and the urban heat island. Atmos. Environ. 1973, 7, 769-779. 
3. Ching, J.K.S. A perspective on urban canopy layer modeling for weather, climate and air quality applications. Urban. Clim. 2013, 3, 13-39.

4. Satterthwaite, A.D.E.; Aragón-Durand, F.; Corfee-Morlot, J.; Kiunsi, M.; Pelling, M.; Roberts, D.C.; Solecki, W. Climate Change 2014: Impacts, Adaptation, and Vulnerability. Part A: Global and Sectoral Aspects. Contribution of Working Group II to the Fifth Assessment Report of the Intergovernmental Panel on Climate Change. Available online: http://www.ipcc.ch/pdf/ assessment-report/ar5/wg2/WGIIAR5-FrontMatterA_FINAL.pdf (accessed on 4 December 2014).

5. Weng, Q. Global Urban Monitoring and Assessment through Earth Observation; CRC Press: Boca Raton, FL, USA, 2014.

6. Esch, T.; Thiel, M.; Schenk, A.; Roth, A.; Muller, A.; Dech, S. Delineation of urban footprints from Terrasar-X data by analyzing speckle characteristics and intensity information. IEEE Trans. Geosci. Remote Sens. 2010, 48, 905-916.

7. Gamba, P.; Aldrighi, M.; Stasolla, M. Robust extraction of urban area extents in HR and VHR SAR images. IEEE J. Sel. Top. Appl. Earth Obs. Remote Sens. 2011, 4, 27-34.

8. Esch, T.; Marconcini, M.; Felbier, A.; Roth, A.; Heldens, W.; Huber, M.; Schwinger, M.; Taubenbock, H.; Muller, A.; Dech, S. Urban footprint processor-Fully automated processing chain generating settlement masks from global data of the TanDEM-X mission. IEEE Geosci. Remote Sens. Lett. 2013, 10, 1617-1621.

9. Miyazaki, H.; Shao, X.; Iwao, K.; Shibasaki, R. An automated method for global urban area mapping by integrating ASTER satellite images and GIS data. IEEE J. Sel. Top. Appl. Earth Obs. Remote Sens. 2013, 6, 1004-1019.

10. Pesaresi, M.; Guo, H.; Blaes, X.; Ehrlich, D.; Ferri, S.; Gueguen, L.; Halkia, M.; Kauffmann, M.; Kemper, T.; Lu, L. A Global Human Settlement Layer from optical HR/VHR RS data: Concept and first results. IEEE J. Sel. Top. Appl. Earth Obs. Remote Sens. 2013, 6, 2102-2131.

11. Ching, J.; Mills, G.; Feddema, J.J.; Oleson, K.; See, L.; Stewart, I.D.; Bechtel, B.; Chen, F.; Wang, X.; Neophytou, M.K.A.; et al. WUDAPT: Facilitating Advanced Urban Canopy Modeling for Weather, Climate and Air Quality Applications. Available online: https://ams.confex.com/ams/ 94Annual/webprogram/Paper236443.html (accessed on 4 December 2014).

12. See, L.; Bechtel, B.; Ching, J.K.S.; Feddema, J.J.; Foley, M.; Fritz, S.; Masson, V.; Mills, G.; Perger, C.; Stewart, I.D. Developing a community-based worldwide urban database of morphology and materials using remote sensing and crowdsourcing for improved urban climate/UHI modeling. Lausanne 2015, submitted.

13. Stewart, I.D.; Oke, T.R. Local climate zones for urban temperature studies. Bull. Am. Meteorol. Soc. 2012, 93, 1879-1900.

14. Bechtel, B.; Daneke, C. Classification of local climate zones based on multiple earth observation data. IEEE J. Sel. Top. Appl. Earth Obs. Remote Sens. 2012, 5, 1191 -1202.

15. Stewart, I.D. A systematic review and scientific critique of methodology in modern urban heat island literature. Int. J. Climatol. 2011, 31, 200-217.

16. Ndetto, E.L.; Matzarakis, A. Urban Atmospheric Environment and Human Biometeorological Studies in Dar es Salaam, Tanzania. Available online: http://link.springer.com/article/ 10.1007/s11869-014-0261-z (accessed on 4 December 2014). 
17. Fenner, D.; Meier, F.; Scherer, D.; Polze, A. Spatial and temporal air temperature variability in Berlin, Germany, during the years 2001-2010. Urban. Clim. 2014, 10, 308-331.

18. Stewart, I.D.; Oke, T.R.; Krayenhoff, E.S. Evaluation of the "local climate zone" scheme using temperature observations and model simulations. Int. J. Climatol. 2014, 34, 1062-1080.

19. Alexander, P.J.; Mills, G. Local climate classification and Dublin's urban heat island. Atmosphere 2014, 5, 755-774.

20. Lelovics, E.; Unger, J.; Gl, T.; Gl, C.V. Design of an urban monitoring network based on local climate zone mapping and temperature pattern modelling. Clim. Res. 2014, 60, 51-62.

21. Gamba, P.; Lisini, G.; Liu, P.; Du, P.; Lin, H. Urban climate zone detection and discrimination using object-based analysis of VHR scenes. In Proceedings of the 4th GEOBIA, Rio de Janeiro, Brazil, 7-9 May 2012.

22. Wurm, M.; Taubenbock, H.; Roth, A.; Dech, S. Urban structuring using multisensoral remote sensing data: By the example of the German cities Cologne and Dresden. In Proceedings of Urban Remote Sensing Event, 2009 Joint, Shanghai, China, 20-22 May 2009; pp. 1-8.

23. Heiden, U.; Heldens, W.; Roessner, S.; Segl, K.; Esch, T.; Mueller, A. Urban structure type characterization using hyperspectral remote sensing and height information. Landsc. Urban Plan. 2012, 105, 361-375.

24. Walde, I.; Hese, S.; Berger, C.; Schmullius, C. From land cover-graphs to urban structure types. Int. J. Geogr. Inf. Sci. 2014, 28, 584-609.

25. Breiman, L. Random forests. Mach. Learn. 2001, 45, 5-32.

26. Weng, Q. Thermal infrared remote sensing for urban climate and environmental studies: Methods, applications, and trends. ISPRS J. Photogramm. Remote Sens. 2009, 64, 335-344.

27. Wulder, M.A.; Masek, J.G.; Cohen, W.B.; Loveland, T.R.; Woodcock, C.E. Opening the archive: How free data has enabled the science and monitoring promise of Landsat. Remote Sens. Environ. 2012, 122, 2-10.

28. Huttner, S.; Bruse, M.; Dostal, P. Using ENVI-Met to Simulate the Impact of Global Warming on the Microclimate in Central European Cities. Available online: http://envi-met.net/documents/ papers/Huttner_etal_2008.pdf (accessed on 4 December 2014).

29. Chen, F.; Kusaka, H.; Bornstein, R.; Ching, J.; Grimmond, C.S.B.; Grossman-Clarke, S.; Loridan, T.; Manning, K.W.; Martilli, A.; Miao, S.; et al. The integrated WRF/urban modelling system: Development, evaluation, and applications to urban environmental problems. Int. J. Climatol. 2011, 31, 273-288.

30. Jackson, T.L.; Feddema, J.J.; Oleson, K.W.; Bonan, G.B.; Bauer, J.T. Parameterization of urban characteristics for global climate modeling. Ann. Assoc. Am. Geogr. 2010, 100, 848-865.

31. Song, C.; Woodcock, C.E.; Seto, K.C.; Lenney, M.P.; Macomber, S.A. Classification and change detection using Landsat TM data: When and how to correct atmospheric effects? Remote Sens. Environ. 2001, 75, 230-244.

32. Knight, E.J.; Kvaran, G. Landsat-8 operational land imager design, characterization and performance. Remote Sens. 2014, 6, 10286-10305. 
33. Conrad, O. SAGA. Aufbau, Funktionsweise und Anwendung eines Systems für geowissenschaftiche Analysen; Göttingen, 2006. Available online: http://ediss.uni-goettingen.de/bitstream/handle/ 11858/00-1735-0000-0006-B26C-6/conrad.pdf?sequence=1 (accessed on 4 December 2014).

34. Köthe, U. Generische Programmierung für die Bildverarbeitung; BoD-Books on Demand: Hamburg, Germany, 2000.

35. Gorelick, N. Google Earth Engine. Available online: http://adsabs.harvard.edu/abs/2012AGUFM. U31A.04G (accessed on 4 December 2014).

36. Van der Kwast, J.; Van de Voorde, T.; Canters, F.; Uljee, I.; Van Looy, S.; Engelen, G. Inferring urban land use using the optimised spatial reclassification kernel. Environ. Model. Softw. 2011, 26, 1279-1288.

37. Blaschke, T. Object based image analysis for remote sensing. ISPRS J. Photogramm. Remote Sens. 2010, 65, 2-16.

38. Ching, J.; Brown, M.; McPherson, T.; Burian, S.; Chen, F.; Cionco, R.; Hanna, A.; Hultgren, T.; Sailor, D.; Taha, H.; et al. National urban database and access portal tool. Bull. Am. Meteorol. Soc. 2009, 90, 1157-1168.

(C) 2015 by the authors; licensee MDPI, Basel, Switzerland. This article is an open access article distributed under the terms and conditions of the Creative Commons Attribution license (http://creativecommons.org/licenses/by/4.0/). 